\title{
DOSSIER
}

\section{Enseñar y aprender en contextos de exclusión ${ }^{1}$ Teaching and Learning in Contexts of Exclusion}

\author{
Sylvia Schmelkes \\ VICERRECTORA ACADÉMICA \\ Universidad Iberoamericana Ciudad de México, México \\ s.schmelkes@ibero.mx \\ https://doi.org/10.48102/didac.2021..77_ENE-JUN.54
}

8

\begin{abstract}
RESUMEN
El artículo expone los retos a los que se enfrenta la escuela mexicana en contextos de exclusión, donde un porcentaje significativo de la población no cuenta con las condiciones elementales para su integración en procesos educativos. Asimismo, se presentan las causas de la exclusión educativa y se ofrecen datos vigentes al respecto. Finalmente, se realiza una reflexión sobre el lugar que debe ocupar el servicio social como relación educativa mutuamente enriquecedora, en la que sus participantes, conscientes de estos contextos de exclusión, propician el crecimiento de las personas y la búsqueda de un mayor bienestar para todos.
\end{abstract}

Palabras clave: Servicio social; exclusión educativa; interacción.

\begin{abstract}
The article exposes the challenges faced by the Mexican school in contexts of exclusion, where a significant percentage of the population does not have the basic conditions to become integrated into educational processes. Likewise, the causes of educational exclusion are presented and current data are offered in this regard. Finally, a reflection is made in regards to the place that social services should occupy as a mutually enriching educational relationship, in which its participants, aware of these contexts of exclusion, promote the growth of people and the search for greater well-being for all.

Keywords: Social Service; Educational Exclusion; Interaction.
\end{abstract}

Fecha de recepción: 18/11/2020

Fecha de aceptación: 23/11/2020

\section{Introducción}

Entiendo al servicio social como una relación educativa que resulta mutuamente enriquecedora. Parto de la premisa de que quien más aprende a través de una relación de apoyo y de compromiso, como la que se da durante el servicio social, es el sujeto que lleva a cabo dicho servicio. De acuerdo con la teoría y las propuestas metodológicas de aprendizaje-servicio (Eyler y Gi-

les, 1999; Rovira y Casares, 2006), el servicio es una oportunidad excepcional para aprender. Igualmente, sabemos la gran valía que tiene aprender en circunstancias de contacto con sujetos y comunidades en situaciones de necesidad o marginalidad, donde también hemos podido aplicar en estos contextos las teorías del aprendizaje situado de Lave y Wenger (2003) y de Díaz Barriga (2003). Al ir del aula al campo, se poten-

100 - Enseñar y aprender en contextos de exclusión Sylvia Schmelkes. DIDAC 77 (2021): 100-106 
cializa el aprendizaje, pues hay una intención transformadora en el servicio social por medio de la praxis, como lo planteó Freire (1982).

\section{¿Por qué lo llamamos "servicio" social?}

Se parte del supuesto de que la formación profesional que reciben los estudiantes en las universidades los equipa para aportar en la solución de problemas, o bien, para contribuir a que crezcan y se desarrollen comunidades. Igualmente, se espera que se retribuya algo de lo recibido en la educación superior. No obstante, esto representa una condición de privilegio para una minoría, pues en América Latina alrededor del $40 \%$, de un grupo de edad entre 18 y 23 años, asiste a alguna institución de educación superior, por lo que la mayoría no llega al grado en el que se debe prestar un servicio social. ${ }^{2}$

Lo nombramos así porque, en menor medida, hay organizaciones y colectivos que, en efecto, requieren de un servicio que no pueden pagar. Pero debemos preguntarnos, ¿cómo podemos asegurar que la relación resulte mutuamente enriquecedora?

Voy a centrar mi mirada a esta relación en el terreno de la educación, de la enseñanza y del aprendizaje, el área del servicio social, la cual no sólo involucra a estudiantes de educación, sino de prácticamente todas las carreras universitarias.

Considero que en ese sentido de reciprocidad, es decir, de esta relación mutuamente enriquecedora, se encuentra justamente lo educativo. Así, educar es facilitar que el otro crezca en autonomía. Todo servicio social que implique contacto con otros tendrá que perseguir y conseguir que el otro crezca en autonomía. Y, en consecuencia, crecerá quien realiza el servicio social.

Vivir es aprender. Se aprende durante la vida. Nunca se deja de aprender, porque crecer es aprender (Ferreiro, 1998). Todos hemos aprendido y tenemos saberes y saberes haceres como consecuencia de nuestro transcurso por la vida. Incluso los niños de preescolar saben cosas; y desde la pedagogía sabemos lo efectivo que es partir de lo que ellos saben y creen. Afortunadamente, conocemos cosas distintas gracias a los diferentes contextos en los que nos desenvolvemos; y es por esto por lo que nos podemos educar unos a otros. Una actitud fundamental será reconocer que el otro tiene saberes y saberes haceres, incluso sabiduría, lo cual es primordial para acercarnos a cualquier proceso de servicio social.

\section{La importancia de la escuela}

Es evidente que hay cosas que se aprenden mejor en la escuela. Ésa es la razón por la que existe el derecho a la educación que, si bien se refiere al derecho a asistir a la escuela, en estricto sentido debe entenderse como el derecho a aprender. Aunque es cierto que todos podemos aprender cualquier cosa —el potencial de aprendizaje es infinito-, las condiciones del contexto limitan este potencial. La pandemia, sin duda, ha restringido el potencial de aprendizaje escolar de niños, niñas y jóvenes y, de manera particular, de los más pobres.

La educación formal conduce al logro de aprendizajes que, de otra forma, resultaría difícil de conseguir, pues es necesario contar con una metodología sistemática (pedagogía) que implica la introducción gradual de demandas intelectuales que conllevan al desarrollo de habilidades superiores de pensamiento. Para que esto ocurra, es crucial el dominio de los lenguajes. Si bien es cierto que se aprende a escuchar y a hablar a través de mecanismos informales, ambos se perfeccionan en la escuela. Asimismo, la lectura de comprensión y la escritura se desarrollan mejor dentro del ámbito escolar, o bien, en programas de educación no formal que facilitan de manera sistemática el aprendizaje.

Por su parte, la formalización de las cuatro operaciones del lenguaje matemático también se concreta a través de procesos sistemáticos y graduados. No obstante, la habilidad para resolver problemas matemáticos propios de contextos específicos puede alcanzarse de manera informal cuando el acceso a la educación es negado o cuando la enseñanza no toma en cuenta el entorno (Carraher \& Carraher, 1985; Ferreiro,1983; Delprato \& Fuenlabrada, 2008). Sin embargo, la formalización en la resolución de problemas matemáticos por medio de algoritmos para sumar, restar, multiplicar y dividir tiene un mejor efecto en la escuela (o mediante programas de edu- 
cación no formal); y lo mismo sucede con las matemáticas superiores. Esto implica trabajar con objetos y sustancias, con maquinaria y equipo; se aprende en laboratorios y talleres, es decir, dentro de ámbitos escolares, o en el lugar de trabajo.

Igualmente, el ámbito escolar se instaura como el entorno privilegiado para aprender a convivir. Amplía el espectro de la diversidad al interactuar con compañeros de diversas procedencias y edades, así como con adultos. Asimismo, facilita el desarrollo personal e integral mediante el aprendizaje cooperativo y el trabajo en equipo para la solución de problemas (aprendizaje basado en proyectos). También, de forma especial, es un lugar privilegiado en el que se aprende a participar democráticamente en la toma de decisiones y en la resolución de conflictos sin recurrir a la violencia. En la escuela, incluso, se puede aprender a tener responsabilidad social sobre otros. Todos estos aprendizajes forjan la estructura valoral de los estudiantes y forman parte de los propósitos de socialización de la escuela. Pese a que no se obtienen con facilidad fuera del ambiente escolar, es cierto que tampoco se aprenden en todas las escuelas.

En este sentido, la escuela se convierte en un lugar propicio para aprender sobre los derechos humanos y sobre cómo defenderlos (tanto los propios como los ajenos). Igualmente, es ahí donde se forja la identidad, la autoestima y un sentido de pertenencia que va más allá de la familia y de la comunidad inmediata.

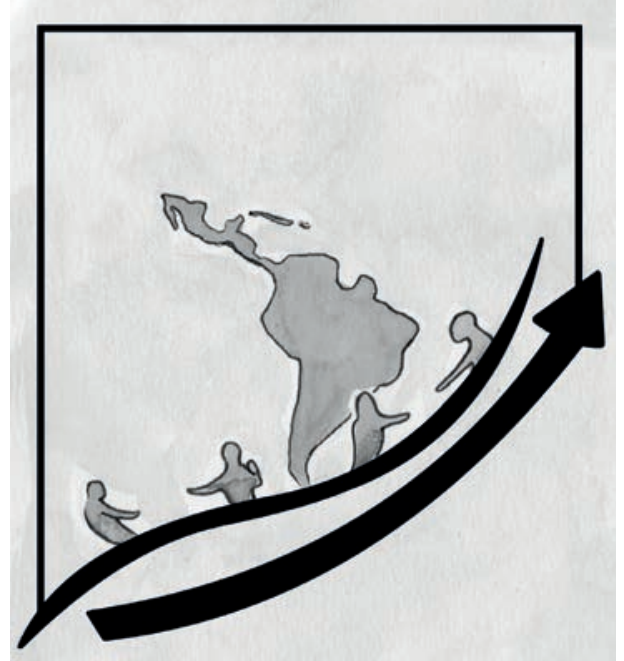

Por último, en ésta se desarrollan las habilidades superiores de pensamiento: el pensamiento abstracto y el pensamiento crítico, los cuales son indispensables para alcanzar niveles superiores de desarrollo moral, que también se adquiere en la escuela y que es clave para la participación democrática responsable, para la acción ciudadana, así como para aprender a respetar a los otros diferentes y consecuentemente, para construir la paz.

Por todo lo anterior, la escuela representa el derecho a una educación de calidad para todos.

\section{Aprender en contextos de exclusión}

La desigualdad en el acceso a la educación es uno de los principales problemas en México. Evidentemente, los más perjudicados son los más pobres, es decir, los que viven en contextos de exclusión. Sabemos quiénes son, cuántos son y por qué tienen conculcado su derecho a aprender lo que se asimila en la escuela.

Los grupos excluidos del derecho a aprender en México son los siguientes:

- Habitantes de zonas rurales menores de 2500 pobladores; sobre todo, localidades a las que no llegan los servicios básicos. En México hay casi 140000 localidades de esta naturaleza, en las que viven alrededor de 650000 niños de entre 3 y 14 ańos (INEGI, 2018). Los niños y las niñas de estos lugares asisten a escuelas multigrado, que en México carecen de un modelo pedagógico y donde los docentes no cuentan con una formación específica que asegure el aprendizaje (INEE, 2019).

- Población en condiciones de pobreza extrema y moderada, que afecta mayormente a los niños y las niñas: 20.7 millones de 0 a 17 años vive en condiciones de pobreza moderada; 5 millones de niños vive en pobreza extrema (Coneval-Unicef, s. f.). Esta situación se concentra, especialmente, en el sector rural.

- Población indígena. México es el país con más indígenas de América: 7 millones de hablantes, 12 millones que viven en poblados indíge-

102 - Enseñar y aprender en contextos de exclusión Sylvia Schmelkes. DIDAC 77 (2021): 100-106 
nas y 25 millones que se autoadscriben como indígenas. Asimismo, es el culturalmente más diverso del continente: 68 grupos etnolingüísticos que hablan 364 variantes de esas lenguas; pero casi $80 \%$ de la población indígena vive en pobreza; por lo tanto, $90 \%$ de los niños y las niñas hablantes de alguna lengua indígena vive en pobreza. La mitad de los indígenas viven en zonas rurales y la otra mitad en zonas urbanas $y$, sin importar el lugar en el que residan, en muchos casos son excluidos de su derecho a aprender (INEE-Unicef, 2015; 2018; 2019; INEGI, 2018).

- Hijos de jornaleros agrícolas migrantes: representan alrededor de un millón y 350000 se encuentra entre los 3 y 17 ańos, edad promedio para cursar la educación básica. Se integran principalmente por indígenas. Los niños, las niñas y los jóvenes migran con sus padres y se emplean desde muy temprana edad en los campos de agricultura extensiva o tecnificada. Sólo $15 \%$ asiste a la escuela (INEE, 2014). Los fondos destinados para su atención, que gravemente venían disminuyendo de manera sistemática, desaparecieron del presupuesto educativo para 2021 al ser aprobado por la Cámara de Diputados (shcP, 2020).

- Población infantil que trabaja: a pesar de que está prohibido por la ley, 3.2 millones de niños y niñas ejerce algún tipo de trabajo. Esto hace que sea el principal enemigo de la escolaridad. Alrededor de 720000 lo hace por más de 20 horas a la semana, muchos de ellos en trabajos de riesgo (INEe, 2018).

- Población con alguna discapacidad: 700000 niños, niñas y jóvenes son atendidos inadecuadamente por la política educativa mexicana, que no ha destinado más que una pequeña oficina a nivel federal para orientar el trabajo educativo en instituciones especializadas o mediante la inclusión en escuelas regulares (INEE, 2018).

En general, estas categorías se yuxtaponen; sin embargo, la mayor (pobreza) coloca al menos a $20 \mathrm{mi}-$ llones de niños en condiciones de exclusión al derecho a aprender.

La población infantil y juvenil que vive en estas condiciones accede menos a la escuela, presenta mayores dificultades en su trayectoria escolar, deserta en mayor porcentaje y aprende menos en este lugar (Gráfica 1). Asimismo, es probable que la falta de relevancia de la educación en esta población provoque que lo aprendido en la escuela no le sirva para tener una vida digna. Sobra decir que la desigualdad educativa se ha exacerbado con la pandemia.

\section{Causas de la exclusión educativa}

Es compleja la etiología de la exclusión educativa. Arrastramos una condición de exclusión histórica que no se contrarresta con una política educativa afirmativa. Esto puede observarse en cómo se distribuyen los recursos tanto financieros como humanos y materiales. En este sentido, se requiere de un sistema educativo que reconozca las causas de la exclusión educativa y que sea capaz de compensarlas, es decir, que atienda más y mejor a los que menos tienen y más necesitan. Desgraciadamente, se ha hecho lo contrario desde que se terminó la primera época de la Escuela Rural Mexicana.

De no contar con una política de igualdad, se continuará dando más recursos a quienes se encuentran en condiciones de relativa ventaja y menos a quienes están en desventaja, esto es, sin asegurar los mismos recursos per cápita para todos nuestros estudiantes. Esta inequitativa distribución se observa en la infraestructura educativa, en el equipamiento del mobiliario, en el material didáctico, en el apoyo brindado al magisterio y en el diferente funcionamiento ordinario de las escuelas (apertura cotidiana, puntualidad y asistencia tanto de docentes como de estudiantes); así como en un solo modelo de escuela que se va empobreciendo en la medida en que llega a poblaciones que no fueron contempladas en su diseño (pequeñas comunidades, pueblos indígenas, hijos de jornaleros agrícolas migrantes, por mencionar algunos), y para las cuales se requiere un modelo escolar específico y de calidad. 


\section{Gráfica 1 \\ Resultados de la prueba Planea en Lenguaje y Comunicación, 2015}

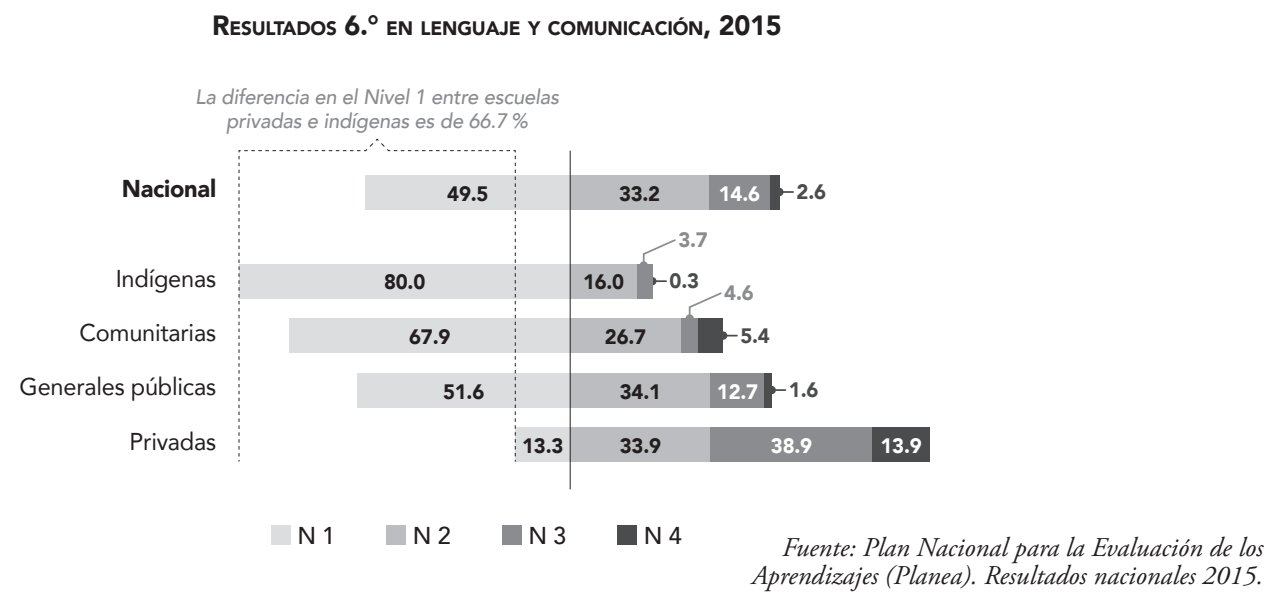

Otro motivo fundamental de exclusión educativa radica en la deficiente enseńanza que se imparte en contextos de pobreza. El currículum en México es único y se aplica en una realidad muy diversa y heterogénea, por lo que responde sólo a las condiciones e intereses de un sector de la población, pero inadecuadamente para muchos otros. La falta de relevancia del aprendizaje también puede observarse en el escaso uso de la lengua materna como lengua de enseñanza en los grupos indígenas; esto se debe a que muchos docentes que laboran en escuelas indígenas no hablan la lengua de la comunidad en la que trabajan.

Pedagógicamente, la falta de relevancia del aprendizaje (que en última instancia, éste se traduce en el valor que el estudiante le otorga para su vida actual y futura) acentúa esta circunstancia por el uso frecuente de la metodología de enseñanza que tiende a privilegiar la entrega de conocimientos por encima del desarrollo de habilidades, y exige a los estudiantes la capacidad de memoria para ver reflejado lo aprendido en una calificación.

La discriminación y el racismo constituyen otra de las causas de la pérdida de significado del aprendizaje en realidades multiculturales, como las zonas urbanas y, fundamentalmente, en sus áreas marginadas o en los campamentos agrícolas. Desafortunadamente, esta situación es poco atendida aún con las medidas de convivencia reguladas en las escuelas.
En suma, estas tres causas se combinan sinérgicamente para afectar el derecho a la educación y al aprendizaje, así como para acentuar la exclusión educativa.

\section{Algunas reflexiones para que el servicio social constituya una experiencia profundamente educativa para las partes implicadas}

\section{Conocer, indignarse, comprometerse}

Antes de emprender el servicio social, es recomendable que estudiantes y profesores conozcan la realidad descrita anteriormente, tanto la expositiva como la causal. De manera ideal, este conocimiento, de naturaleza racional, debería conducir a los estudiantes a indignarse por la realidad y por sus causas, así como por lo inadecuado, por ejemplo, la ausencia de políticas públicas orientadas a atender estas carencias. Así, la indignación es una condición para el compromiso, que a su vez es requisito para que un servicio social resulte mutuamente provechoso.

La verdadera indignación tiene cabida en la interacción con la realidad y, sobre todo, con las personas. Sin embargo, también debe derivarse de un conocimiento científico de la realidad para que la acción se enfoque en la atención de las causas.

\section{Un enfoque intercultural}

Todo encuentro con los otros, sobre todo con los otros diferentes, es un encuentro intercultural, por- 
que al interactuar con el contexto (distinto a nosotros) le otorgamos un sentido a nuestra vida y esto es la esencia de la cultura. Por esto, las culturas que están dentro de una relación de servicio social están en una posición distinta, de ahí que la relación sea mutuamente enriquecedora. Así, el enfoque intercultural supone una absoluta horizontalidad en la relación, éste es esencial para el servicio social, ya que implica el total rechazo a cualquier actitud de superioridad por parte de quien presta el servicio, además, requiere del explícito reconocimiento del valor que tiene toda persona y toda cultura. Asimismo, se debe tener la convicción de que debido a esta diferencia el estudiante se verá enriquecido con dicha relación.

El enfoque intercultural se fundamenta en la filosofía de la alteridad, de la otredad, y sostiene que el otro puede y debe crecer desde lo que es sin solicitarle que borre su diferencia. En este tenor, la filosofía de la otredad supondrá estar convencido de que, precisamente debido a esta diferencia, el estudiante se verá enriquecido al establecer esta relación de servicio. Por último, deberá comprender que las culturas cambian con el tiempo y que son dinámicas, así como el valor de la multiplicidad de culturas de nuestro país y del planeta, pues esto es lo que asegura la vida.

$\mathrm{Al}$ respecto, sería más que conveniente que los estudiantes se formaran desde un enfoque intercultural como paso previo al inicio de esta relación.

\section{Reflexionar, objetivar, metaconocer}

Todo servicio social o voluntariado es una experiencia fuerte, pues es un contacto profundo con el otro, con los otros diferentes. Esta experiencia debe ser una relación de humildad para poner a disposición lo que se sabe y lo que se es y así el otro se apropie de lo que sea de su interés y utilidad.

De igual manera, puede describirse como una experiencia fundamentalmente educativa para ambas partes, que se favorece en la medida en que haya momentos de reflexión sobre lo aprendido, con la finalidad de objetivar lo vivido. Asimismo, esta experiencia sirve para metaconocer, es decir, pensar en cómo "lo aprendî" y "cómo me implica"; de ser posible, externarlo con el otro. Con esto, se promueve que el otro haga lo mismo y lo externe a otros.

\section{Vuelta al comienzo}

Para concluir regreso al inicio: todo servicio social, aunque no esté específicamente orientado a la educación, es educativo per se; aunque el primero con mayor agudeza. Por lo tanto, la interacción debe propiciar el crecimiento de las personas, las habilidades de pensamiento y de juicio moral, a su vez, debe mejorar las condiciones de convivencia y fomentar un mayor bienestar.

En suma, la valoración de la experiencia debe centrarse en "a quién sirvió" y "para qué sirvió". II

REFERENCIAS

Carraher, T. N. \& Carraher, D. W. (1985). En la vida diez, en la escuela cero. México: Siglo XXI Editores.

Consejo Nacional de Evaluación de la Política de Desarrollo Social- Fondo de las Naciones Unidas para la Infancia (Coneval-Unicef). (s. f.). Pobreza infantil y adolescente en México 2008-2016. Recuperado de https://www.coneval.org. $\mathrm{mx} /$ Medicion/Documents/UNICEF_CONEVAL_POBREZA_INFANTIL.pdf

Delprato, M. F. \& Fuenlabrada, I. (2008). Así le hacemos nosotras. Prácticas de numeración escrita en organizaciones productivas de mujeres con baja escolaridad. Cuadernos de Educación, $V I(6)$.

Díaz Barriga, F. (2003). Cognición situada y estrategias para el aprendizaje significativo. Revista Electrónica de Investigación Educativa, 5(2), 1-13.

Eyler, J. \& De Giles, Jr. (1999). Where is the Learning in Service-Learning? San Francisco: Jossey-Bass.
Ferreiro, E. (1983). Los adultos no-alfabetizados y sus conceptualizaciones del sistema de escritura. México: Departamento de Investigaciones Educativas, Centro de Investigación y Estudios Avanzados del IPN.

Ferreiro, E. (1998). Caperucita Roja aprende a escribir. Estudios lingüisticos comparativos en tres lenguas. México: Gedisa.

Freire, P. (1982). La educación como práctica de la libertad. México: Siglo XXI Editores.

Instituto Nacional de Estadística y Geografía (INEGI). (2018). Encuesta Nacional de Ingresos y Gastos de los Hogares 2018. México: INEGI. Recuperado de https://www.inegi.org.mx/ programas/enigh/nc/2018/

Instituto Nacional para la Evaluación de la Educación (INEE). (2014). Evaluación externa de los servicios educativos dirigidos a los niños de familias de jornaleros migrantes. México: INEE/UNPE. Recuperado de https://www.inee.edu.mx/portalweb/suplemento12/evaluacion-servicios-educativos-jornaleros-agricolas-migrantes.pdf

Enseñar y aprender en contextos de exclusión 
Instituto Nacional para la Evaluación de la Educación (INEE). (2015). Plan Nacional para la Evaluación de los Aprendizajes (Planea). Resultados nacionales 2015. 6. ${ }^{\circ}$ de primaria y 3. ${ }^{\circ}$ de secundaria. Lenguaje y Comunicación-Matemáticas. México: INEE. Recuperado de https:/historico.mejoredu.gob.mx/ wp-content/uploads/2019/02/resultadosPlanea-30 11-2015.pdf

Instituto Nacional para la Evaluación de la Educación (INEE). (2018). La educación obligatoria en México. Informe 2018. México: INEE. Recuperado de https://www.inee.edu.mx/ publicaciones/la-educacion-obligatoria-en-mexico-informe-2018/

Instituto Nacional para la Evaluación de la Educación (INEE). (2019). La educación multigrado en México. México: IneE. Recuperado de https:/www.inee.edu.mx/wp-content/ uploads/2019/01/La-Educacio\%CC\%81n-Multigrado_ BIS.pdf

Instituto Nacional para la Evaluación de la Educación-Fondo de las Naciones Unidas para la Infancia (INEE-Unicef). (2015). Panorama educativo de la población indígena. México: INEE-Unicef. Recuperado de https://www.inee.edu.mx/ publicaciones/panorama-educativo-de-la-poblacion-indigena-y-afrodescendiente-2017/

Instituto Nacional para la Evaluación de la Educación-Fondo de las Naciones Unidas para la Infancia (INEE-Unicef).
(2018). Panorama educativo de la población indigena y afrodescendiente. México: INEE-Unicef. Recuperado de https:// www.inee.edu.mx/publicaciones/panorama-educativo-dela-poblacion-indigena-y-afrodescendiente-2017/

Instituto Nacional para la Evaluación de la Educación-Fondo de las Naciones Unidas para la Infancia (INEE-Unicef). (2019). Panorama educativo estatal de la población indígena. Entorno nacional. México: INEE-Unicef. Recuperado de https://www.inee.edu.mx/wp-content/uploads/2019/08/ P3B112.pdf

Lave, J. \& Wenger, E. (2003). Aprendizaje situado. Participación periférica legitima. México: UnAM.

Organización para la Cooperación y el Desarrollo Económicos (OCDE). (2019). Higher Education in Mexico: Labour Market Relevance and Outcomes, Higher Education, París: OCDE Publishing. Recuperado de https://read.oecd-ilibrary.org/ education/higher-education-in-mexico_9789264 309432-en\#page33

Rodríguez, J. P \& Rovira, J. M. R. (2006). Rasgos pedagógicos del aprendizaje-servicio. Cuadernos de Pedagogía, 357, 60-63.

Secretaría de Hacienda y Crédito Público (sHCP). (2020). Proyecto de Decreto de Presupuesto de Egresos de la Federación para el Ejercicio Fiscal de 2021. México: SHCP. Recuperado de https://www.ppef.hacienda.gob.mx/work/models/PPEF 2021 paquete/egresos/Proyecto_Decreto.pdf

\section{SEmblanza}

Socióloga y maestra en Investigación y Desarrollo Educativo por la Universidad Iberoamericana. Realizó estudios de doctorado en Sociología de la Educación en el Instituto de Educación de la Universidad de Londres. En 2008, recibió la medalla Joan Amos

Comenius, otorgada por la República Checa y por la Unesco. En junio de 2019 le otorgaron el doctorado honoris causa por la Université Concordia, en Quebec; y en 2017, por la Universidad Autónoma de Baja California. Es investigadora de la educación desde 1970. Ha publicado más de 150 trabajos, entre libros y artículos, sobre temas de calidad en la educación, educación de adultos, formación en valores y educación intercultural. Es miembro del Sistema Nacional de Investigadores (SNI) desde hace 26 ańos. En la actualidad, cuenta con el reconocimiento en el nivel sNi III. Fundó y coordinó la Coordinación General de Educación Intercultural y Bilingüe en la Secretaría de Educación Pública (20012007). Fungió como presidenta de la Junta de Gobierno del Centro de Investigación e Innovación Educativa de la OCDE (2002-2004). Dirigió el Instituto de Investigaciones para el Desarrollo de la Educación de la Universidad Iberoamericana Ciudad de México de 2007 a 2013. Fue la primera presidenta del INEe de abril de 2013 a abril de 2017; continuó como consejera de su Junta de Gobierno hasta mayo de 2019. Actualmente es vicerrectora académica en la Universidad Iberoamericana Ciudad de México-Tijuana.

\footnotetext{
${ }^{1}$ Conferencia presentada en el 37 Congreso Nacional y 11 Internacional de Servicio Social y Voluntariado Universitario, y IV Coloquio Interamericano sobre el Voluntariado Universitario, organizados por la Comisión Interuniversitaria del Servicio Social (ciss), a través del Centro Intercultural de Reflexión y Acción Social (ciras), de la Universidad Iberoamericana Puebla, el 12 de octubre de 2020.

${ }^{2}$ La población mexicana entre 24 y 65 años con un título de educación superior es de apenas $17 \%$, por debajo de otros países como Chile (23\%), Colombia (23\%), Costa Rica (23\%) y Argentina (21\%) (oCDE, 2019).
} 